\title{
TOPOLOGY AND GEOMETRY OF RANDOM 2-DIMENSIONAL HYPERTREES
}

\author{
MATTHEW KAHLE AND ANDREW NEWMAN
}

\begin{abstract}
AвstRAct. A hypertree, or $\mathbb{Q}$-acyclic complex, is a higher-dimensional analogue of a tree. We study random 2-dimensional hypertrees according to the determinantal measure suggested by Lyons. We are especially interested in their topological and geometric properties. We show that with high probability, a random 2-dimensional hypertree $T$ is apsherical, i.e. that it has a contractible universal cover. We also show that with high probability the fundamental group $\pi_{1}(T)$ is hyperbolic and has cohomological dimension 2 .
\end{abstract}

\section{InTRODUCTION}

The following enumerative formula is well known.

Theorem 1. The number of spanning trees on $n$ vertices is

$$
n^{n-2} \text {. }
$$

The trees are understood to be labelled, i.e. on vertex set $[n]:=\{1,2, \ldots, n\}$, and not merely up to isomorphism type. The example $n=4$ is illustrated in Figure 1. There are only 2 trees on 4 vertices up to isomorphism, but there are 16 labelled trees.

Apparently, Theorem 1 was first proved by Borchardt in 1860 [8]. Cayley extended the statement in 1889 [10], and it is often known as "Cayley's formula." Several proofs can be found in Aigner and Ziegler's book [1]. Aigner and Ziegler write that the "most beautiful proof of all" was given by Avron and Dershowitz [5], based on ideas of Pitman.

The definition of a tree is that it is connected and has no cycles. Equivalently, a graph $G$ is a tree if it has no nontrivial homology, i.e. if $\tilde{H}_{0}(G)=H_{1}(G)=0$. Kalai suggested the topological notion of $\mathbb{Q}$-acyclic simplicial complexes as higher-dimensional analogues of trees in [22]. $\mathbb{Q}$-acyclic complexes are sometimes called hypertrees. Here, we use the term 2-tree for a 2-dimensional hypertree. The precise definition is as follows.

Definition 2. We say that a finite 2-dimensional simplicial complex $S$ is a 2-tree if it has all of the following properties.

- $S$ has complete 1-skeleton, i.e. if the underlying graph is a complete graph.

- $H_{1}(S ; \mathbb{Q})=H_{2}(S ; \mathbb{Q})=0$.

Kalai proved a general formula for a weighted enumeration of $\mathbb{Q}$-acyclic complexes, which specializes to the following in the case of 2-trees.

Date: April 29, 2020.

M.K. was supported in part by NSF-CCF grants \#1740761 and \#1839358. He is grateful to the Simons Foundation for a Simons Fellowship, and to the Berlin Mathematical School for a Mercator Fellowship.

A.N. was supported by Deutsche Forschungsgemeinschaft (DFG, German Research Foundation) Graduiertenkolleg 2434 "Facets of Complexity". 


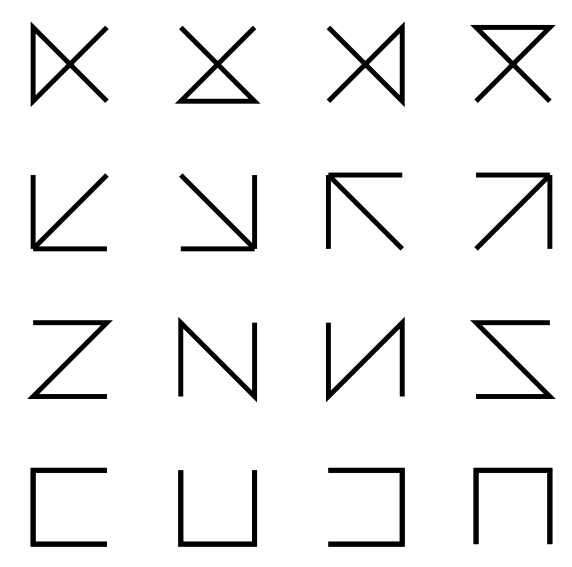

Figure 1 . The $4^{2}=16$ trees on 4 vertices.

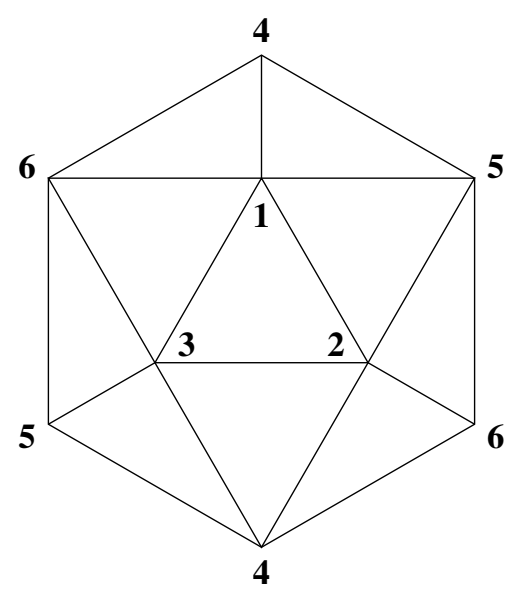

FIGURE 2. The smallest topologically nontrivial 2-tree is the 6-vertex projective plane.

Theorem 3 (Kalai [22]).

$$
\sum_{S \in \mathcal{T}(n)}\left|H_{1}(S)\right|^{2}=n^{\left(\begin{array}{c}
n-2 \\
2
\end{array}\right)}
$$

Here the notation $|G|$ denotes the order of the group $G$. Since $H_{1}(S ; \mathbb{Q})=0$ by definition, by the universal coefficient theorem we have that $H_{1}(S)$ is a finite group for every $S \in \mathcal{T}(n)$.

The smallest topologically nontrivial example of a 2-tree is the 6-vertex projective plane, illustrated in Figure 2. A topological space is said to be aspherical if it has a contractible universal cover. The 6-vertex projective plane is a good example to show that 2-trees are not always aspherical.

More general enumerative formulas were given by Duval, Klivans, and Martin [15]. These generalizations again are weighted enumeration formulas. In fact, currently for $d \geq 2$ establishing the unweighted enumeration for $d$-trees is an open problem. The bestknown upper and lower bounds on unweighted enumeration are given by Linial and Peled [26]. 
Kalai's enumeration suggests a natural probability distribution on 2-trees, first studied by Lyons [29]. Let $\mathcal{T}(n)$ denote the set of all 2 -trees on vertex set $[n]$. Define a probability measure on $\mathcal{T}(n)$ by making the probability of every 2 -tree $T$ proportional to $\left|H_{1}(T)\right|^{2}$. Equivalently, by Kalai's formula, the probability of any particular 2-tree $T$ is given by

$$
\mathbb{P}(T)=\frac{\left|H_{1}(T)\right|^{2}}{n^{\left(\begin{array}{c}
n-2 \\
2
\end{array}\right)}} .
$$

This is the distribution we study for the rest of this paper. This distribution is in many ways nicer than the uniform distribution. The most important property of this probability distribution for our applications is that it satisfies negative association. This is an a result of Lyons [29] that we review in Section 2.

We write $T \sim \mathcal{T}(n)$ to denote a 2 -tree chosen according to the determinantal measure described above. For any property $P_{n}$, we say that property $P_{n}$ occurs with high probability (w.h.p.) if $\mathbb{P}\left[T \in P_{n}\right] \rightarrow 1$ as $n \rightarrow \infty$. We are mostly interested in topological and geometric properties of $T$. Our main results are that w.h.p., $T$ is aspherical and that $\pi_{1}(T)$ is a hyperbolic group of cohomological dimension 2 . The proofs depend on combining ideas from probability, topology, and geometric group theory.

We note that many other models of random simplicial complex have been studied see, for example, the survey in Chapter 22 of [17]. The closest model to what we study here is the Linial-Meshulam model $Y \sim Y(n, p)$ introduced in [25], which is the "faceindependent" model, a higher-dimensional analogue of the Erdős-Rényi edge-independent random graph $G(n, p)$. Negative association allows us to relate random 2-trees with $Y(n, p)$. Babson, Hoffman, and Kahle showed the fundamental group $\pi_{1}(Y)$ (in a certain range of parameter) is hyperbolic, with high probability, and Costa and Farber showed that $Y$ is "almost" aspherical, and they also showed that $\pi_{1}(Y)$ has cohomological dimension 2.

The remainder of the paper is organized as follows. In Section 2, we review definitions of "determinantal measures" and negative association. In Section 3, we show that w.h.p. the fundamental group of the random 2-tree $\pi_{1}(T)$ is a hyperbolic group w.h.p. In Section 4 we show that w.h.p. $H_{1}(T) \neq 0$ and in Section 5 we show that w.h.p. $\pi_{1}(T)$ is cohomologically 2-dimensional. In Section 6 , we suggest a few questions for future study.

\section{Negative association}

We first review the definitions of determinantal measure and negative association. In particular, we briefly overview the work of Lyons [28, 29] which is essential for our results. In [28], Lyons defines a determinantal probability measure as follows.

Definition 4. Given a finite set $E$, a probability measure $\mu$ on $E$ is said to be a determinantal probability measure if there exists a matrix $M$ so that for all $S \subseteq E$, the probability that a subset $T$ sampled by $\mu$ contains $S$ as a subset is given by $\operatorname{det}\left(M_{S, S}\right)$, i.e. the determinant of the submatrix of $M$ whose rows and columns are indexed by $S$.

A monotone increasing event is an event $\mathcal{A}$ so that $S \in \mathcal{A}$ and $S \subseteq T$ implies that $T \in \mathcal{A}$. A key fact about determinantal measure is that they satisfy negative association, defined in [29] as follows.

Definition 5. Given a finite set $E$, a probability measure $\mu$ on $E$ is said to satisfy negative association provided that for every pair of monotone increasing events $\mathcal{A}$ and $\mathcal{B}$

$$
\mu(\mathcal{A} \cap \mathcal{B}) \leq \mu(\mathcal{A}) \mu(\mathcal{B}) .
$$


Lyons shows in Theorem 6.5 of [28] that determinantal probability measures satisfy negative association, and in [29] that the torsion-squared distribution on 2-trees we consider here is a determinantal measure.

For our purposes here, we will primarily be interested in the situation where we wish to bound the probability that a determinantal-measure sampled 2-tree contains some particular, finite subcomplex. In our case, given $n$, the set $E$ in the definition of a determinantal probability measure is the set of all $\left(\begin{array}{l}n \\ 3\end{array}\right)$ triangles on $n$ vertices. By Euler characteristic any 2-tree contains exactly $\left(\begin{array}{c}n-1 \\ 2\end{array}\right)$ triangles, so we have by symmetry that under the torsionsquared distribution the probability that a random 2-tree contains any particular face is

$$
\frac{\left(\begin{array}{c}
n-1 \\
2
\end{array}\right)}{\left(\begin{array}{c}
n \\
3
\end{array}\right)}=\frac{3}{n}
$$

Thus, for a fixed (labeled) subcomplex $K$ given by triangles $\sigma_{1}, \ldots, \sigma_{k}$, we have by negative association that the probability that $T$ sampled from the torsion-squared distribution contains $K$ as a subcomplex is at most $(3 / n)^{k}$. For this reason we say that the faces of a torsionsquared random 2 -tree are negatively correlated.

In contrast to the determinantal measure, the uniform measure on 2-trees need not have negatively correlated faces. This can be seen by a exhaustive enumeration of 2-trees on 6 vertices. This is discussed in [21], and we review the discussion as follows. There are 46620 2 -trees on vertex set $\{1, \ldots, 6\}$. As 2 -trees on 6 vertices contain $\left(\begin{array}{l}5 \\ 2\end{array}\right)=10$ triangles out of a total of $\left(\begin{array}{l}6 \\ 3\end{array}\right)=20$ possible triangles, by symmetry we have that the probability that a uniform random 2 -tree contains any given triangle is $10 / 20=1 / 2$. On the other hand, 116642 trees contain both the triangle $[1,2,3]$ and the triangle $[4,5,6]$ by exhaustive enumeration. However 11664/46620 $>1 / 4$. Changing to the torsion-squared distribution resolves this in the case $n=6$ because 12 of the 2-trees on 6 vertices are labeled triangulations of the projective plane. None of these contain both $[1,2,3]$ and $[4,5,6]$. Sampling by torsionsquared counts these 12 complexes each 4 times and gives that the probability a 2 -tree contains both $[1,2,3]$ and $[4,5,6]$ is $11664 / 46656=1 / 4$.

\section{HyPERBolicity}

We show in this section that w.h.p. $\pi_{1}(T)$ is hyperbolic in the sense of Gromov [18]. The proof is based on the main result in [6] -indeed, we will use a key lemma from the paper as our main tool. We first review a few key definitions and notions related to hyperbolicity.

Let $C_{r}$ denote a cycle of length $r$. For a simplicial complex $X$, a loop is a simplicial map $\gamma: C_{r} \rightarrow X$. In this case, we define the length of $\gamma$ by $L(\gamma)=r$.

We say that $\left(C_{r} \stackrel{b}{\rightarrow} D \stackrel{\pi}{\rightarrow} X\right)$ is a filling of $\gamma$ if $D$ is a simplicial complex, $b$ and $\pi$ are simplicial maps such that $\gamma=\pi b$, and the mapping cylinder of $b$ is homeomorphic to a 2-dimensional disk.

Let $f_{2}(D)$ denote the number of 2-dimensional faces in $D$. We define the area of the filling to be the number of faces in $f_{2}(D)$. For a null-homotopic loop $\gamma$, we say that the area of $\gamma$, denoted $A(\gamma)$, is the minimal area over all fillings.

Now, we are ready for a definition of hyperbolic group.

Definition 6. Let $\Delta$ be a finite simplicial complex. We say that the fundamental group $\pi_{1}(\Delta)$ is hyperbolic if there exists a constant $K>0$ such that

$$
A(\gamma) \leq K L(\gamma)
$$

for every null-homotopic loop $\gamma$. 


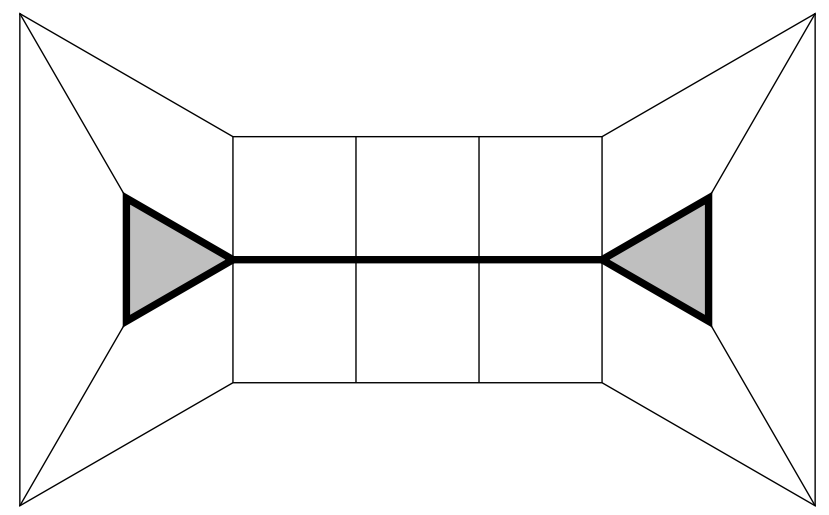

FIgURE 3. The mapping cylinder of $b$ for a filling $\left(C_{r} \stackrel{b}{\rightarrow} D \stackrel{\pi}{\rightarrow} X\right)$ of a cycle $\gamma$. Here $L(\gamma)=12$ and $A(\gamma)=2$.

It is not obvious from this definition, but this is an invariant property of the group $\pi_{1}(\Delta)$ which does not depend on the choice of simplicial complex $\Delta$. This definition in terms of a linear isoperimetric inequality is similar to the first definition given by Gromov in [18]. Satisfying such an inequality is equivalent to a Cayley graph of the group being $\delta$-hyperbolic, or the group being word hyperbolic.

Our main tool in this section is the following, which appears in [6].

Theorem 7 (Babson-Hoffman-Kahle, Theorem 1.9 in [6]). Let $\epsilon>0$, and suppose that $\Delta$ is a finite simplicial complex such that for every subcomplex $S \subseteq \Delta$, we have that

$$
\frac{f_{2}(S)}{f_{0}(S)} \leq 2-\epsilon .
$$

Then $\Delta$ satisfies a linear isoperimetric inequality. Namely

$$
A(\gamma) \leq \lambda L(\gamma)
$$

for every null-homotopic loop $\gamma$. Here $\lambda=\lambda(\epsilon)$ is a constant which only depends on $\epsilon$.

We also require the following, which allows us to pass from local to global isoperimetric inequalities. This particular statement for simplicial complexes and its proof also appear in [6], and it is based on earlier work of Gromov [18] and Papasoglu [31].

Theorem 8. Suppose that $\rho \geq 1$ and $X$ is a finite simplicial complex for which every null-homotopic loop $\gamma: C_{r} \rightarrow X$ with $A(\gamma) \leq 44^{3} \rho^{2}$ satisfies $A(\gamma) \leq \rho L(\gamma)$. Then every null-homotopic loop $\gamma: C_{r} \rightarrow X$ satisfies $A(\gamma) \leq 44 \rho L(\gamma)$.

In other words, if $X$ satisfies a linear isoperimetric inequality locally, then it satisfies one globally, although perhaps with a worse isoperimetric constant. So it suffices to check hyperbolicity on balls of finite radius. We are now ready to prove the main result of the section.

Theorem 9. Suppose $T \sim \mathcal{T}(n)$ is a random 2-tree according to the determinantal measure. Then w.h.p. $\pi_{1}(T)$ is a hyperbolic group.

Proof of Theorem 9. With foresight into the calculations to come, let $\epsilon=1 / 2$, and let $\lambda=\lambda(\epsilon)$ be the constant guaranteed by Theorem 7 . So for every finite simplicial complex 
$\Delta$ satisfying the condition of Theorem 77 and every null-homotopic loop $\gamma: C_{r} \rightarrow \Delta$, we have

$$
A(\gamma) \leq \lambda L(\lambda)
$$

Now, let $C$ be chosen such that

$$
C \geq \max \left\{44^{3} \lambda^{2}, 44^{3}\right\},
$$

and then let $C^{\prime}$ be chosen such that

$$
C^{\prime} \geq \frac{C}{2}\left(1+\frac{1}{\lambda}\right)+1
$$

We emphasize that $C$ and $C^{\prime}$ are chosen to be sufficiently large, but are still fixed as $n \rightarrow \infty$.

First, we check that w.h.p. for every subcomplex $\Delta \subset T$ on at most $C^{\prime}$ vertices, we have

$$
\frac{f_{2}(\Delta)}{f_{0}(\Delta)}<\frac{3}{2}
$$

Note first that if there exists a subcomplex $\Delta \subset T$ with $f_{2}(\Delta) \geq(3 / 2) f_{0}(\Delta)$, then there exists a subcomplex $\Delta^{\prime} \subset T$ with $f_{2}\left(\Delta^{\prime}\right)=\left\lceil(3 / 2) f_{0}\left(\Delta^{\prime}\right)\right\rceil$. Indeed, $\Delta^{\prime}$ can be obtained by deleting one face from $\Delta$ at a time until equality is achieved.

A union bound, together with negative association, gives that

$$
\mathbb{P}\left[\exists \Delta \subset T \text { with } f_{2}(\Delta)>(3 / 2) f_{0}(\Delta)\right] \leq \sum_{k=1}^{C^{\prime}}\left(\begin{array}{l}
n \\
k
\end{array}\right)\left(\begin{array}{c}
\left(\begin{array}{c}
k \\
3
\end{array}\right) \\
\lceil(3 / 2) k\rceil
\end{array}\right)\left(\frac{3}{n}\right)^{\lceil(3 / 2) k\rceil} .
$$

The sum tends to zero as $n$ tends to infinity, since $C^{\prime}$ is fixed so there are only a bounded number of summands, and every summand tends to zero.

By Theorem 7, we have that w.h.p. every subcomplex $\Delta \subset T$ on at most $C^{\prime}$ vertices satisfies the linear isoperimetric inequality

$$
A(\gamma) \leq \lambda L(\gamma) .
$$

Next, we check that this implies that

$$
A(\gamma) \leq \lambda L(\gamma)
$$

for every null-homotopic loop $\gamma$ in $T$ with $A(\gamma) \leq C$.

Suppose that $\gamma$ is a null-homotopic loop with $A(\gamma) \leq C$. If $L(\gamma)>C / \lambda$, then since $A(\gamma) \leq C$ it is immediate that $A(\gamma) \leq \lambda L(\gamma)$.

So suppose instead that $L(\gamma) \leq C / \lambda$. In this case, $A(\gamma)$ and $L(\gamma)$ are both bounded. It follows that if $\left(C_{r} \stackrel{b}{\rightarrow} D \stackrel{\pi}{\rightarrow} T\right)$ is a filling of $\gamma$, then the number of vertices $f_{0}(D)$ is bounded as well. Indeed, let $v, e$, and $f$ denote the number of vertices, edges, and faces in the mapping cylinder of $b$. Since we have a bijection between vertices of the mapping cylinder, and the disjoint union of vertices in $C_{r}$ and vertices in $D$, we have

$$
v=L(\gamma)+f_{0}(D) \text {. }
$$

By double counting edge-face incident pairs have $2 e=5 L+3 A$. or

$$
e=(5 / 2) L+(3 / 2) A \text {. }
$$

Finally, we have

$$
f=L+A,
$$

since every face of the mapping cylinder is either a square face (corresponding to a single edge of $C_{r}$ ) or a triangle face of the simplicial complex $D$. Since the mapping cylinder is a topological disk, we have

$$
v-e+f=1 \text {. }
$$


Putting it all together gives that $f_{0}(D)=A(\gamma) / 2+L(\gamma) / 2+1$.

In the case we are interested in, we have

$$
\begin{aligned}
f_{0}(D) & =A(\gamma) / 2+L(\gamma) / 2+1 \\
& \leq \frac{C}{2}+\frac{C}{2 \lambda}+1 \\
& =\frac{C}{2}\left(1+\frac{1}{\lambda}\right)+1 \\
& \leq C^{\prime}
\end{aligned}
$$

by choice of $C^{\prime}$. Then the image of the map $\pi: D \rightarrow T$ lies in subcomplex $\Delta \subset T$ on at most $C^{\prime}$ vertices, so by the above $A(\gamma) \leq \lambda L(\gamma)$, as desired.

Let $\rho=\max \{1, \lambda\}$. Then $\rho \geq 1$ and we have that $A(\gamma) \leq \rho L(\gamma)$ for every null-homotopic loop $\gamma$ with $A(\gamma) \leq C$. Theorem 8 gives that

$$
A(\gamma) \leq 44 \rho L(\gamma)
$$

for all null-homotopic $\gamma$ in $T$. Setting $K=44 \rho$, we have the desired result.

\section{NONTRIVIALITY AND EXPECTED ORDER OF TORSION}

In this section, we give upper bounds on the probability that homology $H_{1}(T)$ is trivial and lower bounds on its expected order. We make use of the following observation of Kalai [22] on the number of 2-trees on $n$ vertices.

Lemma 10. Let $N(n)$ denote the number of 2-trees on $n$ vertices. Then

$$
N(n) \leq(e n / 3)^{\left(\begin{array}{c}
n-1 \\
2
\end{array}\right)} .
$$

Proof. Every 2-tree $T$ on $n$ vertices has $\left(\begin{array}{l}n \\ 2\end{array}\right)$ edges. The Betti numbers are $\beta_{0}=1$ and $\beta_{1}=\beta_{2}=0$, by definition. By the Euler formula, $T$ has $\left(\begin{array}{c}n-1 \\ 2\end{array}\right) 2$-dimensional faces. So the total number of 2 -trees is at most

$$
\left(\begin{array}{c}
\left(\begin{array}{c}
n \\
3
\end{array}\right) \\
\left(\begin{array}{c}
n-1 \\
2
\end{array}\right)
\end{array}\right) \leq\left(\frac{e\left(\begin{array}{c}
n \\
3
\end{array}\right)}{\left(\begin{array}{c}
n-1 \\
2
\end{array}\right)}\right)^{\left(\begin{array}{c}
n-1 \\
2
\end{array}\right)}=\left(\frac{e n}{3}\right)^{\left(\begin{array}{c}
n-1 \\
2
\end{array}\right)}
$$

Theorem 11. Let $T \in \mathcal{T}(n)$. With probability at least $1-\exp \left(-\Omega\left(n^{2}\right)\right)$, we have $H_{1}(T) \neq 0$.

Proof. The probability that $X$ sampled from $C_{n}$ with respect to the determinantal measure has $H_{1}(X)=0$ is

$$
\begin{aligned}
\sum_{\left\{X \mid H_{1}(X)=0\right\}} \frac{1}{n^{\left(\begin{array}{c}
n-2 \\
2
\end{array}\right)}} & =\frac{\text { Number of 2-trees with } H_{1}(X)=0}{n^{\left(\begin{array}{c}
n-2 \\
2
\end{array}\right)}} \\
& \leq \frac{N(n)}{n^{\left(\begin{array}{c}
n-2 \\
2
\end{array}\right)}}
\end{aligned}
$$

By Lemma 10, we have that the above is at most

$$
\left(\frac{e n}{3}\right)^{\left(\begin{array}{c}
n-1 \\
2
\end{array}\right)} \frac{1}{n^{\left(\begin{array}{c}
n-2 \\
2
\end{array}\right)}}=\left(\frac{e}{3}\right)^{\left(\begin{array}{c}
n-1 \\
2
\end{array}\right)} n^{n-2} .
$$

It follows that the probability that $H_{1}(T)=0$ is $\exp \left(-\Omega\left(n^{2}\right)\right)$.

Next, we prove the following. 
Theorem 12. We have that

$$
\mathbb{E}\left[\left|H_{1}(T)\right|\right] \geq\left(\frac{3}{e}\right)^{\left(\begin{array}{c}
n-2 \\
2
\end{array}\right)}\left(\frac{3}{e n}\right)^{n-2} .
$$

So in particular, we have that

$$
\mathbb{E}\left[\left|H_{1}(T)\right|\right]=\exp \left(\Theta\left(n^{2}\right)\right)
$$

We will use the following inequality.

Lemma 13. Let $x_{1}, x_{2}, \ldots x_{k} \geq 0$ be non-negative real numbers.

Then it follows that

$$
\sum_{i=1}^{k} x_{i}^{3} \geq \frac{1}{\sqrt{k}}\left(\sum_{i=1}^{k} x_{i}^{2}\right)^{3 / 2}
$$

Proof of Lemma 13 Jensen's inequality tells us that for a convex function $\phi$, numbers in its domain $y_{1}, y_{2}, \ldots, y_{k}$, and positive weights $a_{1}, a_{2}, \ldots, a_{k}$, we have

$$
\phi\left(\frac{\sum_{i=1}^{k} a_{i} y_{i}}{\sum_{i=1}^{k} a_{i}}\right) \leq \frac{\sum_{i=1}^{k} a_{i} \phi\left(y_{i}\right)}{\sum_{i=1}^{k} a_{i}} .
$$

Set $a_{i}=1$ and $y_{i}=x_{i}^{2}$ for $i=1,2, \ldots k$, and let $\phi(x)=x^{3 / 2}$. We note that $\phi(x)$ is convex on the domain $\{x \mid x \geq 0\}$.

Given the lemma, we prove Theorem 12 .

Proof of Theorem 12. By definition, we have that

$$
\begin{aligned}
\mathbb{E}\left[\left|H_{1}(T)\right|\right] & =\sum_{T \in \mathcal{T}(n)} \mathbb{P}[T]\left|H_{1}(T)\right| \\
& =\sum_{T \in \mathcal{T}(n)} \frac{\left|H_{1}(T)\right|^{2}}{n^{\left(\begin{array}{c}
n-2 \\
2
\end{array}\right)}}\left|H_{1}(T)\right| \\
& =\frac{1}{n^{\left(\begin{array}{c}
n-2 \\
2
\end{array}\right)}} \sum_{T \in \mathcal{T}(n)}\left|H_{1}(T)\right|^{3} \\
& \geq \frac{n^{3 / 2}\left(\begin{array}{c}
n-2 \\
2
\end{array}\right)}{n^{\left(\begin{array}{c}
n-2 \\
2
\end{array}\right)}(e n / 3)^{(1 / 2)\left(\begin{array}{c}
n-1 \\
2
\end{array}\right)}} .
\end{aligned}
$$

This last step is by applying Lemmas 10 and 13 . Simplifying, we have that

$$
\begin{aligned}
\mathbb{E}\left[\left|H_{1}(T)\right|\right] & \geq\left(\sqrt{\frac{3}{e}}\right)^{\left(\begin{array}{c}
n-2 \\
2
\end{array}\right)}\left(\sqrt{\frac{3}{e n}}\right)^{n-2} \\
& =\left((3 / e)^{1 / 4}-o(1)\right)^{n^{2}} .
\end{aligned}
$$


This is on the scale of the largest torsion possible, in the sense that for every simplicial complex $\Delta$ on $n$ vertices, we have that the order of the torsion part of homology is bounded by

$$
\left|H_{1}(\Delta)_{\text {torsion }}\right| \leq \sqrt{3}^{\left(\begin{array}{c}
n-1 \\
2
\end{array}\right)} \leq\left(3^{1 / 4}-o(1)\right)^{n^{2}},
$$

This upper bound on torsion appears in many places, including [32] and [19], and perhaps first appeared in Kalai's weighted enumeration of hypertrees [22].

\section{5. $T$ IS ASPHERICAL AND $\pi_{1}(T)$ HAS COHOMOLOGICAL DIMENSION 2}

The main result of this section is the following.

Theorem 14. Let $T \sim \mathcal{T}(n)$. Then, w.h.p. $T$ is aspherical.

Our proof will use the following theorem of Costa and Farber [13]. It is worth noting that this is a purely topological and combinatorial statement, and does not involve probability.

Theorem 15 (Costa-Farber, Theorem 11 of [13]). There exists a finite list $\mathcal{L}$ of compact 2-dimensional complexes with the following two properties:

(1) A finite simplicial 2-complex $Y$ is aspherical if it contains no subcomplex isomorphic to a complex from the list $\mathcal{L}$.

(2) For any $S \in \mathcal{L}$ other than the boundary of the tetrahedron, there exists a subcomplex $S^{\prime} \subseteq S$ with $f_{0}\left(S^{\prime}\right) / f_{2}\left(S^{\prime}\right) \leq 46 / 47$

We've modified the statement slightly from its original form. In [13], Costa and Farber show that for a certain regime of $p, Y(n, p)$ is asphericable, that is it has the property that after removing a single face from every embedded tetrahedron boundary the resulting complex is aspherical. In the original formulation, the conclusion of part (1) is that the complex is asphericable. Here we simply added the tetrahedron boundary to the set $\mathcal{L}$ as we already know that a 2 -tree $T$ cannot contain tetrahedron boundaries, since $H_{2}(T ; \mathbb{Q})=0$.

Proof of Theorem 14. Take $\mathcal{L}$ to be the finite list of complexes in Theorem 15 . We show that with high probability $T \sim \mathcal{T}(n)$ contains no subcomplex in $\mathcal{L}$. We already know that $T$ cannot contain the boundary of a tetrahedron; for any other $S \in \mathcal{L}$, we bound the probability that a determinantal-measure random 2-tree contains $S$. For $S \in \mathcal{L}$, different from the tetrahedron boundary, take $S^{\prime}$ to be a subcomplex of $S$ satisfying condition (2) of Theorem 15 and let $v$ denote $f_{0}\left(S^{\prime}\right)$, then the probability that $T \sim \mathcal{T}(n)$ contains $S$ is at most the probability that it contains $S^{\prime}$. By negative correlation the probability that $T$ contains $S^{\prime}$ is at most

$$
\left(\begin{array}{l}
n \\
v
\end{array}\right) v !\left(\frac{3}{n}\right)^{47 v / 46} .
$$

Indeed to embed $S^{\prime}$ in $T$ we have to choose the $v$ vertices and then we have $\left|\operatorname{Aut}\left(S^{\prime}\right)\right| \leq v$ ! ways to choose a copy of $S^{\prime}$ on the selected vertex set. Now by negative correlation the probability that every face of the selected copy of $S^{\prime}$ appears in $T$ is at most the product of the probability that each face of $S^{\prime}$ appears, thus it is at most $(3 / n)^{f\left(S^{\prime}\right)} \leq(3 / n)^{47 v / 46}$. As $v$ is fixed and at least one the probability that $T$ contains $S^{\prime}$ as an embedded subcomplex is $O\left(n^{-1 / 46}\right)$. By a union bound over the finite list $\mathcal{L}$, the probability that $T$ contains any member of $\mathcal{L}$ is $O\left(n^{-1 / 46}\right)=o(1)$. Thus by part (1) of Theorem 15 , with probability at least $1-O\left(n^{-1 / 46}\right), T \sim \mathcal{T}(n)$ is aspherical.

For a group $G$, let $\operatorname{cd}_{R}(G)$ denote the cohomological dimension of $G$ with respect to coefficient ring $R$. We have the following immediate consequence of Theorem 14 
Theorem 16. Let $T \sim \mathcal{T}(n)$. Then w.h.p. $c d_{\mathbb{Z}}\left(\pi_{1}(T)\right)=2$.

Proof. In Section 4 , we saw that w.h.p. $H_{1}(T) \neq 0$ is nontrivial. By definition, $H_{1}(T, \mathbb{Q})=0$, so we have that w.h.p. $H_{1}(T)$ is a nontrivial group, and not a free group. By the StallingsSwan Theorem [33, 34], we have $\operatorname{cd}_{\mathbb{Z}}\left(\pi_{1}(T)\right) \geq 2$.

On the other hand, if $T$ is aspherical then $T$ is itself a 2-dimensional $B G$ for $G=\pi_{1}(T)$, so $\operatorname{cd}_{\mathbb{Z}}\left(\pi_{1}(T)\right) \leq 2$.

We end with a comment. If $\operatorname{cd}_{\mathbb{Z}}\left(\pi_{1}(T)\right)=2$, then w.h.p. $\pi_{1}(T)$ must be infinite. Indeed, w.h.p. $\pi_{1}(T)$ is a nontrivial group, and it can not have any elements of finite order, since this would imply that the cohomological dimension is infinite. Even though our results show that (according to the determinantal measure) almost all hypertrees $T$ have infinite fundamental group $\pi_{1}(T)$, at the moment we are not aware of any explicit examples.

\section{Questions}

It seems to us that the random 2-tree is a natural model for stochastic topology. We suggest a few more questions for further study.

- Does $\pi_{1}(T)$ have Kazhdan's Property (T)? A group is said to have Property (T) if the trivial representation is an isolated point in the unitary dual equipped with the Fell topology. This is an important property in representation theory, geometric group theory, ergodic theory, and the theory of expander graphs. See the monograph [7] for a comprehensive introduction. We conjecture that for $T \sim \mathcal{T}(n)$, w.h.p. $\pi_{1}(T)$ has Property (T). One motivation for the conjecture is that in [20], it is shown that in the stochastic process version of the Linial-Meshulam random 2-complex, as soon as the complex $Y$ is pure 2-dimensional, $\pi_{1}(Y)$ has Property (T). In general, it would be interesting to know about "high-dimensional" expander properties of random 2-trees. See Lubotzky's 2018 ICM talk for an overview of high-dimensional expanders [27].

- Is $H_{1}(T)$ Cohen-Lenstra distributed? Cohen-Lenstra heuristics, first arising in number-theoretic settings [12], are a natural model for random finite abeliean groups. These heuristics now appear in several contexts, including cokernels of random matrices and random graph Laplacians. See, for example, [11, 16, 23, 24, [30, 35]. In [21], Kahle, Lutz, Newman, and Parsons studied the uniform measure on random 2-trees, and examined the random finite abelian groups that appeared as the first homology group. There is strong experimental evidence for the conjecture that for any fixed prime $p$, the probability that the Sylow $p$-subgroup of homology $G$ is distributed according to a probability distribution assigning probability inversely proportional to $|\operatorname{Aut}(G)|$. Equivalently, for a given prime $p$ and $p$-group $H$, the probability that $G$ is isomorphic to $H$ is given by the formula

$$
\frac{\prod_{k=1}^{\infty}\left(1-p^{-k}\right)}{|\operatorname{Aut}(H)|}
$$

We expect this same limiting probability holds, even if the 2-trees are sampled by the determinantal measure instead. One can sample a 2-tree with with the Metropolis-Hastings algorithm, and preliminary experiments support the conjecture. 


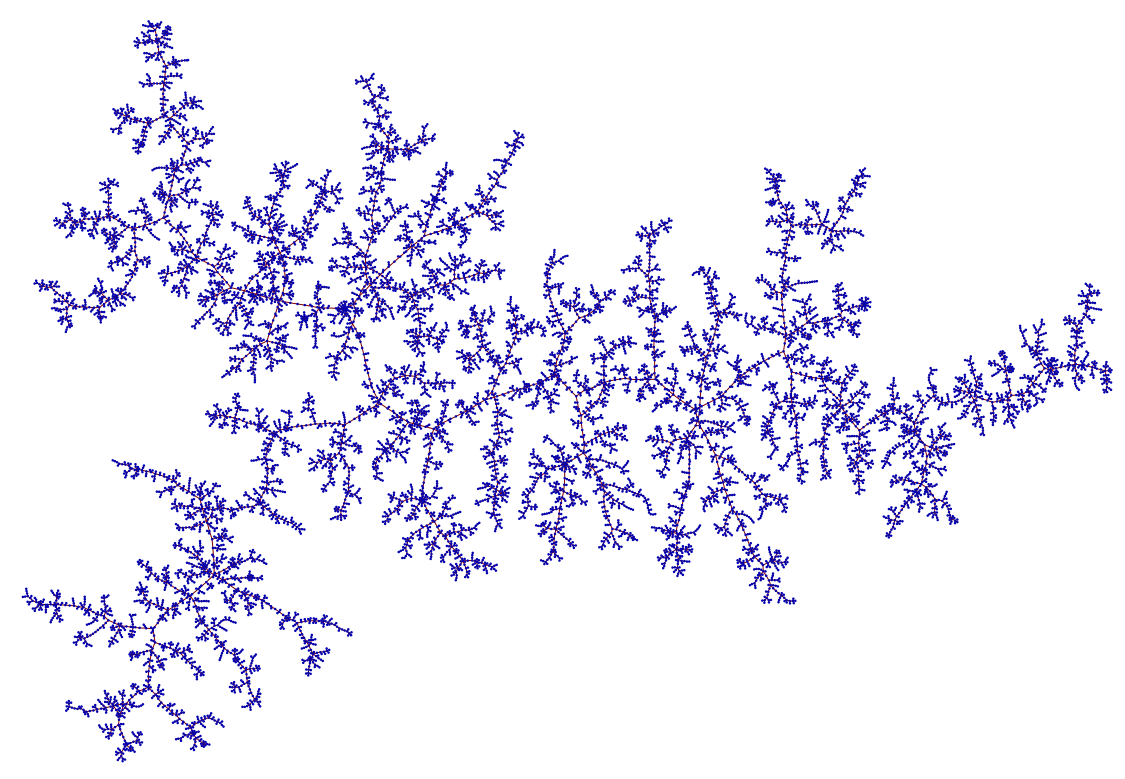

Figure 4. An image of a continuum random tree generated by Igor Kortchemski.

- Is there a scaling limit? The random 2-tree is a 2-dimensional analogue of the uniform spanning tree (UST) on the complete graph on $n$ vertices. The UST is known to have a scaling limit, where a suitably rescaled UST converges to a limiting distribution as $n \rightarrow \infty$. This limit was described by Aldous in [2, 3, 4], who called it the "continuum random tree", and it has been studied extensively since then. An illustration of a continuum random tree computed by by Igor Kortchemski appears in Figure 4 Is there a scaling limit for the random 2-tree?

\section{ACKNOWLEDGEMENTS.}

M.K. is grateful to Nati Linial for suggesting the study of random hypertrees and for encouragement. We thank TU Berlin for hosting us during the 2019-20 academic year. We also gratefully acknowledge Igor Kortchemski's permission for use of the image in Figure 4.

\section{REFERENCES}

[1] Martin Aigner and Günter M. Ziegler. Proofs from The Book. Springer, Berlin, sixth edition, 2018. See corrected reprint of the 1998 original [ MR1723092], Including illustrations by Karl H. Hofmann.

[2] David Aldous. The continuum random tree. I. Ann. Probab., 19(1):1-28, 1991.

[3] David Aldous. The continuum random tree. II. An overview. In Stochastic analysis (Durham, 1990), volume 167 of London Math. Soc. Lecture Note Ser., pages 23-70. Cambridge Univ. Press, Cambridge, 1991.

[4] David Aldous. The continuum random tree. III. Ann. Probab., 21(1):248-289, 1993.

[5] Arnon Avron and Nachum Dershowitz. Cayley's formula: a page from the book. Amer. Math. Monthly, 123(7):699-700, 2016.

[6] Eric Babson, Christopher Hoffman, and Matthew Kahle. The fundamental group of random 2-complexes. $J$. Amer. Math. Soc., 24(1):1-28, 2011.

[7] Bachir Bekka, Pierre de la Harpe, and Alain Valette. Kazhdan's property (T), volume 11 of New Mathematical Monographs. Cambridge University Press, Cambridge, 2008. 
[8] C. W. Borchardt. Ueber eine der Interpolation entsprechende Darstellung der Eliminations-Resultante. J. Reine Angew. Math., 57:111-121, 1860.

[9] Kenneth S. Brown. Cohomology of groups, volume 87 of Graduate Texts in Mathematics. Springer-Verlag, New York-Berlin, 1982.

[10] Arthur Cayley. A theorem on trees. Quart. J. Pure Appl. Math., 23:376-376, 1889.

[11] J. Clancy, N. Kaplan, T. Leake, S. Payne, and M. M. Wood. On a Cohen-Lenstra heuristic for Jacobians of random graphs. J. Algebraic Combin., 42(3):701-723, 2015.

[12] H. Cohen and H.W. Lenstra. Heuristics on class groups of number fields. Number theory, Noordwijkerhout 1983, 1068:33-62, 1984.

[13] A. E. Costa and M. Farber. The asphericity of random 2-dimensional complexes. Random Structures Algorithms, 46(2):261-273, 2015.

[14] A. E. Costa and M. Farber. Geometry and topology of random 2-complexes. Israel J. Math., 209(2):883-927, 2015.

[15] Art M. Duval, Caroline J. Klivans, and Jeremy L. Martin. Simplicial matrix-tree theorems. Trans. Amer. Math. Soc., 361(11):6073-6114, 2009.

[16] J. S. Ellenberg, A. Venkatesh, and C. Westerland. Homological stability for Hurwitz spaces and the CohenLenstra conjecture over function fields. Ann. of Math. (2), 183(3):729-786, 2016.

[17] Jacob E. Goodman, Joseph O'Rourke, and Csaba D. Tóth, editors. Handbook of discrete and computational geometry. Discrete Mathematics and its Applications (Boca Raton). CRC Press, Boca Raton, FL, 2018. Third edition of [ MR1730156]

[18] M. Gromov. Hyperbolic groups. In Essays in group theory, volume 8 of Math. Sci. Res. Inst. Publ., pages 75-263. Springer, New York, 1987.

[19] Christopher Hoffman, Matthew Kahle, and Elliot Paquette. The threshold for integer homology in random $d$-complexes. Discrete Comput. Geom., 57(4):810-823, 2017.

[20] Christopher Hoffman, Matthew Kahle, and Elliot Paquette. Spectral gaps of random graphs and applications. International Mathematics Research Notices (IMRN), 05 2019. https://doi.org/10.1093/imrn/rnz077.

[21] Matthew Kahle, Frank H. Lutz, Andrew Newman, and Kyle Parsons. Cohen-Lenstra heuristics for torsion in homology of random complexes. Exp. Math., 2018. https://doi.org/10.1080/10586458.2018.1473821.

[22] G. Kalai. Enumeration of Q-acyclic simplicial complexes. Israel J. Math., 45(4):337-351, 1983.

[23] S. Koplewitz. Sandpile groups and the coeulerian property for random directed graphs. Advances in Applied Mathematics, 90:145 - 159, 2017.

[24] J. Lengler. The global Cohen-Lenstra heuristic. Journal of Algebra, 357(Supplement C):347 - 369, 2012.

[25] Nathan Linial and Roy Meshulam. Homological connectivity of random 2-complexes. Combinatorica, 26(4):475-487, 2006.

[26] Nati Linial and Yuval Peled. Enumeration and randomized constructions of hypertrees. Random Structures Algorithms, 55(3):677-695, 2019.

[27] Alexander Lubotzky. High dimensional expanders. In Proceedings of the International Congress of Mathematicians-Rio de Janeiro 2018. Vol. I. Plenary lectures, pages 705-730. World Sci. Publ., Hackensack, NJ, 2018.

[28] Russell Lyons. Determinantal probability measures. Publ. Math. Inst. Hautes Études Sci., (98):167-212, 2003

[29] Russell Lyons. Random complexes and $l^{2}$-Betti numbers. J. Topol. Anal., 1(2):153-175, 2009.

[30] Hoi H. Nguyen and Melanie Matchett Wood. Random integral matrices: universality of surjectivity and the cokernel. arXiv:1806.00596, 2018.

[31] P. Papasoglu. An algorithm detecting hyperbolicity. In Geometric and computational perspectives on infinite groups (Minneapolis, MN and New Brunswick, NJ, 1994), volume 25 of DIMACS Ser. Discrete Math. Theoret. Comput. Sci., pages 193-200. Amer. Math. Soc., Providence, RI, 1996.

[32] C. Soulé. Perfect forms and the Vandiver conjecture. J. Reine Angew. Math., 517:209-221, 1999.

[33] John R. Stallings. On torsion-free groups with infinitely many ends. Ann. of Math. (2), 88:312-334, 1968.

[34] Richard G. Swan. Groups of cohomological dimension one. J. Algebra, 12:585-610, 1969.

[35] Melanie Matchett Wood. The distribution of sandpile groups of random graphs. J. Amer. Math. Soc., 30(4):915-958, 2017. 
(M.K.) The Ohio State University, 100 Math Tower, 231 W 18th Ave, Columbus, OH 43210

E-mail address: mkahle@math.osu.edu

(A.N.) Technische Universität Berlin, Chair of Discrete Mathematics / Geometry, Strasse des 17. Juni 136, 10623 Berlin, Germany

E-mail address: newman@math.tu-berlin.de 